\title{
ACCESS - a NASA mission concept study of an Actively-Corrected Coronagraph for Exoplanet System Studies
}

\author{
John Trauger ${ }^{\mathrm{a}}$, Karl Stapelfeldt ${ }^{\mathrm{a}}$, Wesley Traub ${ }^{\mathrm{a}}$, Curt Henry $^{\mathrm{a}}$, John Krist ${ }^{\mathrm{a}}$ \\ Dimitri Mawet $^{\mathrm{a}}$, Dwight Moody ${ }^{\mathrm{a}}$, Peggy Park ${ }^{\mathrm{a}}$, Laurent Pueyo ${ }^{\mathrm{a}}$, Eugene Serabyn ${ }^{\mathrm{a}}$ \\ Stuart Shaklan $^{\mathrm{a}}$, Olivier Guyon ${ }^{\mathrm{b}, \mathrm{c}}$, Jeremy Kasdin ${ }^{\mathrm{d}}$, David Spergel ${ }^{\mathrm{d}}$, Robert Vanderbei ${ }^{\mathrm{d}}$ \\ Ruslan Belikov, Geoff Marcy ${ }^{\mathrm{f}}$, Robert A. Brown ${ }^{\mathrm{g}}$, Jean Schneider ${ }^{\mathrm{h}}$ \\ Bruce Woodgate ${ }^{\mathrm{i}}$, Gary Matthews ${ }^{\mathrm{j}}$, Robert Egerman ${ }^{\mathrm{j}}$, Ronald Polidan ${ }^{\mathrm{k}}$ \\ Chuck Lillie $^{\mathrm{k}}$, Mark Ealey ${ }^{\mathrm{m}}$, Thomas Price ${ }^{\mathrm{m}}$ \\ ${ }^{a}$ Jet Propulsion Laboratory,California Institute of Technology, Pasadena, CA 91109 \\ ${ }^{\mathrm{b}}$ Subaru Telescope, Hilo, HI 96720 \\ ${ }^{\mathrm{C} U n i v e r s i t y ~ o f ~ A r i z o n a, ~ T u c s o n, ~ A Z ~} 85721$ \\ ${ }^{\mathrm{d}}$ Princeton University, Princeton, NJ 08540 \\ ${ }^{\mathrm{e}}$ NASA Ames Research Center, Moffett Field, CA 94035 \\ ${ }^{f}$ University of California, Berkeley, CA 94720 \\ ${ }^{\mathrm{g}}$ Space Telescope Science Institute, Baltimore, MD 21218 \\ ${ }^{\text {h}}$ Paris Observatory, Meudon, France \\ ${ }^{\mathrm{i}}$ NASA Goddard Space Flight Center, Greenbelt, MD 20771 \\ ${ }^{\mathrm{j}}$ ITT Space Systems Division, Rochester, NY 14650 \\ ${ }^{\mathrm{k}}$ Northrop Grumman Corporation, Redondo Beach, CA 90278 \\ ${ }^{\mathrm{m}}$ Xinetics - Northrop Grumman, Devens, MA 01434
}

\begin{abstract}
ACCESS (Actively-Corrected Coronagraph for Exoplanet System Studies) develops the science and engineering case for an investigation of exosolar giant planets, super-earths, exo-earths, and dust/debris fields that would be accessible to a medium-scale NASA mission. The study begins with the observation that coronagraph architectures of all types (other than the external occulter) call for an exceptionally stable telescope and spacecraft, as well as active wavefront correction with one or more deformable mirrors (DMs). During the study, the Lyot, shaped pupil, PIAA, and a number of other coronagraph architectures will all be evaluated on a level playing field that considers science capability (including contrast at the inner working angle (IWA), throughput efficiency, and spectral bandwidth), engineering readiness (including maturity of technology, instrument complexity, and sensitivity to wavefront errors), and mission cost so that a preferred coronagraph architecture can be selected and developed for a medium-class mission.
\end{abstract}

Keywords: coronagraphs, exoplanets, space astronomy, active optics

\section{INTRODUCTION}

The goal of this mission is to open a portal to the discovery and characterizations of nearby planetary systems. Our objective is to select and develop the optimal medium-class coronagraph mission for the imaging and spectroscopy of exoplanetary systems, based on a cost-benefit analysis of the known coronagraph architectures. We expect mature planetary systems (such as our own) to be a dynamic mix of planets large and small with debris fields created by collisions between planetesimals. For the nearby stars that are key targets in the search for Earth-like planets, a mediumclass coronagraphic mission will reveal the location, density, and extent of dust clouds, down to the sub-zodi level; spectrally characterize giant planets discovered by radial velocity surveys; and search for outer giant planets not yet accessible to radial velocity (RV) detection. We have a special interest in the "habitable zone", the distance from a star at which stellar radiation raises surface temperatures into the range for liquid water. Depending on the limiting coronagraphic performance achieved and the specific exozodiacal background levels, a medium-class mission has the potential to detect an Earth-like planet around as many as a dozen nearby stars. Finally, this mission will provide a high contrast imaging capability that can be applied to astrophysical problems, such as the evolution of dust structures surrounding pre- and post- main sequence stars, spanning the life cycle of planetary systems. 


\section{SCIENCE OBJECTIVES}

\subsection{Direct imaging in the context of diverse investigations from the ground and space}

The architecture of exoplanetary systems, including planets and debris structures, lies beyond the reach of conventional techniques. The proposed exoplanet and zodi targets are too faint and too close to the parent star to be observable by current observatories on the ground or in space. Transit and micro-lensing studies capture rare "lucky" orbital alignments, providing a statistical census of planets orbiting distant stars, but are unlikely to detect more than a single planet in any given planetary system. RV and astrometry techniques are insensitive to dust structures, hence will not identify asteroid belts nor characterize the exozodiacal background levels. Eclipse methods are sensitive only to close-in "roaster" planets. The contrast performance of large ground-based telescopes with adaptive optics currently falls four orders of magnitude short of what is needed to image exoplanets or tenuous exozodiacal dust in reflected light around nearby stars. Next generation AO systems and ELTs are expected to make up about two orders of magnitude this shortfall in contrast.

ACCESS is designed to reveal planets and dust structures by reducing the scattered and diffracted light within a few arcseconds of a star to a level three orders of magnitude lower than any current instrument in space or on the ground, using a stable 1.5 meter diameter telescope, a coronagraphic system for control of diffracted light, active wavefront correction and suppression of scattered light, and a low-noise CCD focal plane and integral field spectrograph viewing the target systems in multiple spectral bands simultaneously.

\subsection{Zodi structure as an indicator of unseen planets}

ACCESS will make a sensitive imaging survey of tenuous debris disks, the signposts of planetary systems. These are clouds of small dust grains created by the mutual collisions of asteroids and comets, as well as by comet sublimation. Without continual replenishment by such events, the dust would be cleared away within a small fraction of a star's lifetime by stellar radiation, stellar wind pressure, or self-radiation. The interplanetary dust cloud in our own solar system, which can be seen as the zodiacal light, is a debris disk created primarily by the collisions of asteroids. Spitzer observations have identified 40 stars within $25 \mathrm{pc}$ that possess debris disk analogs to our Kuiper Belt, at the levels of a few hundred zodis. Some of these dust clouds have been spatially resolved and show warps, asymmetries, or narrow rings that are strongly suggestive of planetary perturbations. The Earth has resonantly trapped dust within a ring along its orbit, with a trailing wake of enhanced particle density ${ }^{1}$; the Fomalhaut debris disk has a significant eccentricity that requires dynamical forcing by a planet of at least Neptune mass ${ }^{2}$. As a canvas on which planets can impress dynamical signatures, dust disk structures will enable indirect detections of planets too faint or dust-shrouded to otherwise be seen.

\subsection{Probe of Zodi dust as a potential problem for planet detection}

High levels of exozodiacal dust will be a major impediment to direct imaging of extrasolar terrestrial planets, no matter what telescope architecture is eventually employed. These dust clouds reflect starlight and emit in the thermal infrared, adding photon noise and scene confusion to exoplanet signals. If typical exozodiacal dust levels proved to be an order of magnitude greater than our solar system's, it would force the TPF mission design to high spatial resolutions that could only be accomplished with a very large telescope/coronagraph or a formation-flying interferometer. It is therefore crucial to assess the dust levels around nearby stars. Spitzer 70-micron observations have identified exo-Kuiper disks brighter than 100 zodis in spatially unresolved photometry; Herschel may improve on this sensitivity by a factor of 2 . However, these observations detect cold dust far from the habitable zone (HZ). The Keck and LBT interferometers will use 10-micron nulling observations to probe HZ dust. However, initial Keck observations have shown that the combination of atmospheric and instrumental phase stability limits the null depth to sensitivities of roughly a hundred zodis. Additional ways to attack the problem are needed. Detailed coronagraphic imaging simulations show that an observatory like ACCESS can provide sensitivity down to the sub-zodi level, extending inward to $\sim 3 \mathrm{x}$ the $\mathrm{HZ}$ across the likely sample of all TPF targets, and into the HZ itself for about a dozen stars. Transport models can be used to relate dust levels at $3 \mathrm{AU}$ to those at $1 \mathrm{AU}$; for our solar system, these models are well-calibrated by dust counts from interplanetary probes. Reflected light observations with ACCESS will thus be an outstanding tool for assessing the dust content in the HZs of nearby stars.

\subsection{Census of nearby stars and known RV planets beyond $1 \mathrm{AU}$}

Nine nearby stars are currently known to host radial velocity planets whose apastron distances project to angular separations $>0.20$ arcsec. These are cold objects presenting a contrast of $10^{-9}$ in reflected starlight in the optical and near-IR, and thus are only accessible to a space mission. Existing ephemerides allow observations to be timed to coincide with their maximum elongations. In addition, there are six stars within $20 \mathrm{pc}$ that show long-term accelerations,

Proc. of SPIE Vol. 7010 701029-2 
indicating the presence of outer planets with orbits still not yet defined. ACCESS will directly detect these fifteen giant planets, measure their colors, take spectra at $\mathrm{R} \sim 20$, and provide astrometric measurements that resolve the $\sin i$ ambiguity in their masses. The spectra will allow the strength of $\mathrm{CH}_{4}$ features to be measured and the depth of the uppermost cloud deck to be inferred. The planets' albedo and presence of bright ring systems can be inferred using the observed photometry and planet sizes estimated from their measured masses. Multi-epoch imaging showing the planetary orbital motions will make a powerful visual impression of the reality of exoplanetary systems in the public mind.

\subsection{Planet Searches beyond RV Survey Limits}

Radial velocity searches to date have yielded detections of giant planets within 6 AU of FGK stars with low chromospheric activity. Coronagraphic imaging searches with a 1.5-meter telescope are restricted to planets outside a typical inner working angle of $3 \mathrm{AU}$ for stars within $15 \mathrm{pc}$, and to 1 AU for perhaps a dozen targets. ACCESS coronagraphic observations have the potential to discover giant planets at distances as large as $15 \mathrm{AU}$ from their central stars, objects for which the RV signal is low and orbital period very long. It will also be able to detect planets around stars inaccessible to precision RV measurements: active stars that are spectrally noisy, and the A stars which lack a rich absorption spectrum. For a search of the 3-15 AU region, 3 epochs of observations spaced at the beginning, middle, and end of a five year mission will provide a sufficient exploration of orbital phase space to achieve a detection completeness exceeding $70 \%$. For the $\sim$ dozen stars where searches as close as $1 \mathrm{AU}$ are possible, $6-10$ epochs of imaging may be needed to compile good completeness results. There is the potential to detect terrestrial planets in the HZ of these systems, if ACCESS imaging finds low levels of exozodiacal dust.

\subsection{Dust to Dust: the life cycle of planetary systems}

ACCESS will enable sensitive scattered light measurements of the structure of circumstellar matter in a wide-range of astrophysics settings. Many hundreds of young protoplanetary disks are known, but barely a dozen have been imaged at subarcsecond resolution. Only a few bright disks are visible against the glare of their central star ${ }^{3}$; most HST imaging detections to date have taken place in special circumstances of external illumination ${ }^{4}$, edge-on orientation ${ }^{5}$, or large central clearings ${ }^{6}$. The numerous unseen disks have apparently darkened significantly, perhaps because of dust grain evolution or settling which can produce self-shadowing in these optically thick systems ${ }^{7}$. These dimmer, more evolved disks will be revealed around young stellar objects by ACCESS, completing our understanding of disk evolution from the protoplanetary to the debris disk phases.

By making observations of Sun-like stars at the end of their lives, ACCESS will follow the mass-loss which seeds the ISM with processed gas and dust leading to the next generation of stars and planetary systems. Sun-like stars become significantly cooler, larger and more luminous as they traverse the Red Giant Branch (RGB) and then the Asymptotic Giant Branch (AGB) regions of the H-R diagram. The large luminosity increases of an RGB star ( $\left.\sim 30-100 \mathrm{~L}_{\mathrm{SUN}}\right)$ will lead to evaporation and disintegration of pre-existing comets $^{8}$, indirectly indicated by the detection of far-infrared excesses in such stars. Close dust shells in post-main sequence stars will trace the interaction between pulsating stellar winds, residual debris disks, and low mass companions that defines the bewildering variety of structures found in the subsequent planetary nebula phase.

\subsection{Significance of the mission concept}

Given that ACCESS could make the first spectral measurements of reflected light from up to about a dozen RV planets, and quite possibly an equal number of super-earths, the impact on exoplanet science would be huge, because no comparable measurements will exist before then, and there will have been a lack of data with which to confront models. Searching for an analogy, we note that a flurry of papers and a general great interest in transit data, including the presence of water and methane on a planet that is almost certainly not likely to support life ${ }^{9}$, has resulted from a few low-signal-to-noise spectra that have been obtained from Spitzer and HST.

In addition, ACCESS will produce images and spectra of dust disks, which likewise have had very little visiblewavelength data to date, so a similar level of excitement and impact can be anticipated here. We know essentially nothing about zodiacal dust disks at the level of our solar system, so being able to measure many such examples will be an exciting event. These data will lead us back to the collisional sources of dust, the rate of dissipation of dust, and a host of related questions. 


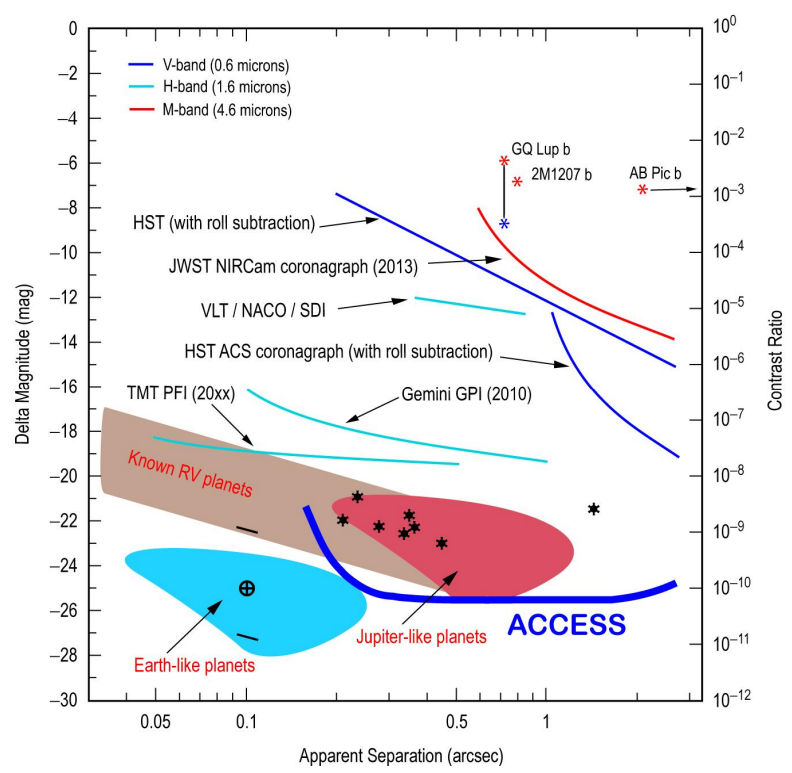

Figure 1. ACCESS imaging sensitivity compared to other methods available now and in the coming decade. Shaded areas indicate the regions of high probability (from Monte-Carlo simulations) of detectable planets orbiting the nearest 100 AFGK stars. Jupiters in 5 AU orbits fall in the red-shaded area, where specific RV-identified planets are shown by asterisks. Earth-like planets fall in the blue-shaded area. The location of an Earth-twin at elongation at $10 \mathrm{pc}$ is indicated by the $\oplus$ symbol, with a dash above for the background from a dust disk similar to the solar system, and a dash below to indicate the level of background noise after one day of integration. The detection range for ACCESS is the area above the bold curve at lower right.

As illustrated in Figure 1, contrasts in the range of $10^{-7}$ to $10^{-8}$, yielding luminous young Jupiters, will be accomplished in the foreseeable future only by very large ground-based systems, assuming that these work as hoped, but the region from $10^{-8}$ to $10^{-10}$, where the mature Jupiters, super-earths, and dust disks reside, will be completely unknown territory until ACCESS or its equivalent flies in space.

It is evident that the exact placement of the ACCESS sensitivity curve in the diagram sets the stage for the science mission. While Jupiter-twins orbiting the nearest 100 AFGK stars fall well within the ACCESS detection zone, Earthtwins and super-earths (up to five times as bright) orbiting the same stars are near the margins. The ACCESS study will provide the best engineering estimates on the achievable inner working angle (which could shift the blue curve to the right or left) and just as important, the level of wavefront control and contrast (which could shift the curve up or down). The ACCESS study provides the venue for an authoritative consensus on the science capabilities of a medium-class mission for imaging and spectroscopy of nearby exoplanet systems.

The relevance of ACCESS to NASA programs would be enormous. We recognize that to fully characterize Earth-like planets, including searching for signs of life on them, we will need significantly larger telescope diameters than possible within the scope of a NASA medium-scale mission. Thus the information from ACCESS will be a valuable precursor for subsequent flagship missions, in terms of science knowledge and technical ability. It is much easier to plan a mission if you know in advance that the technology really works, and that the targets actually exist. ACCESS can supply all this, and thereby enable future NASA missions of even greater scope.

\section{OBSERVATORY ARCHITECTURE}

We define a medium-class observatory that supports the ACCESS science objectives with mature technologies and establishes an initial point design for our study. It includes the following major subsystems: an unobscured 1.5-meter Gregorian telescope, (2) a spacecraft bus configured for operations at L2, (3) a pointing control system to accurately center the star image on the coronagraph focal plane mask, (4) a wavefront control system that includes two precision deformable mirrors in series, and (5) a low-noise imaging sensor and integral-field spectrograph, all rated for a nominal 5 -year science mission. 


\subsection{Design criteria}

We seek high confidence that all critical components of the ACCESS concept will reach TRL 6 or better by the completion of Phase A. This is already the case for the ACCESS observatory, as itemized below. However, the TRLs range widely for components of the various coronagraphs. Demonstrated and anticipated TRLs for the coronagraph components will guide our selection of preferred coronagraph types.

ACCESS will survey the known coronagraph architectures, subject to the engineering realities of the baseline telescope and spacecraft systems. We cover the gamut of known coronagraph types by comparing science performance and engineering tolerances of the following four specific coronagraph methods: (1) amplitude attenuation at the focal plane (band-limited Lyot coronagraph), (2) amplitude attenuation in a pupil plane (shaped pupil coronagraph), (3) phase apodization in the pupil plane (pupil mapping or PIAA), and (4) phase modulation at the focal plane (vortex phase mask coronagraph). While noting that, in practice, all coronagraph types include elements to control both amplitude and phase of the complex optical wavefront, we can classify the coronagraph types by the nature and location of the dominant diffraction control element (Figure 2). A closer look reveals that the best-performing examples of each of these basic coronagraph types, far from being pure and distinct, are more usefully regarded as selected cases among a gamut of hybrid approaches. The ACCESS team will use the "level playing field" provided by its baseline design to explore and quantify the advantages of the hybrid approach to coronagraph design.
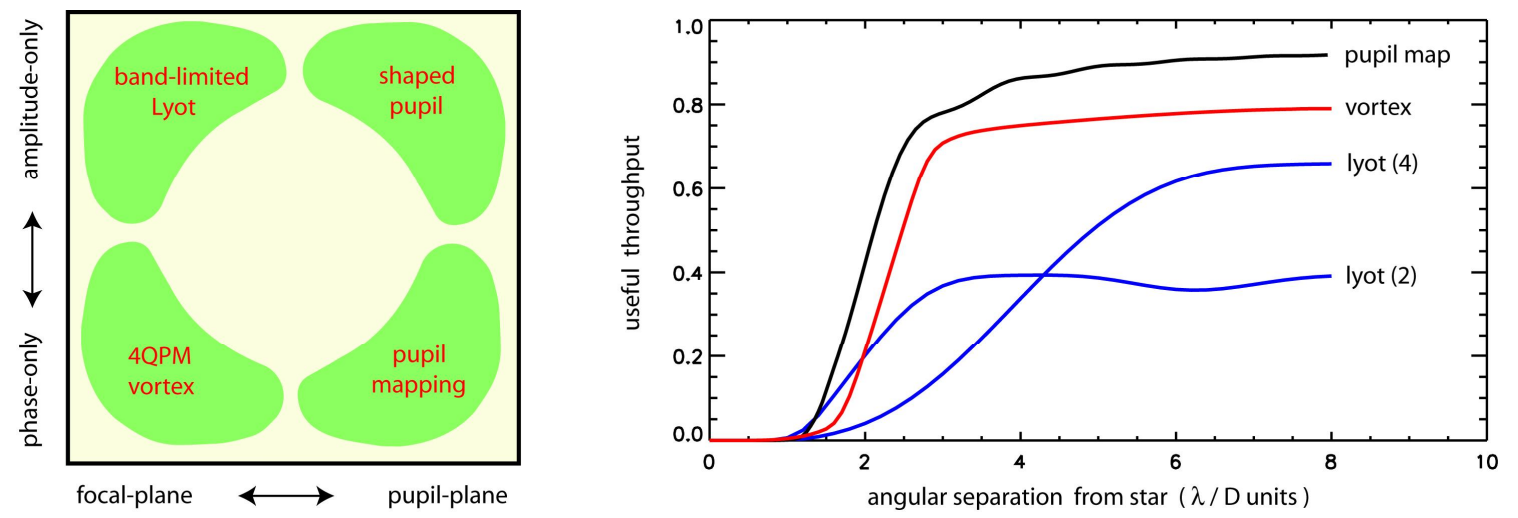

Figure 2. At left: a notional coronagraph design space. At right: Plot of useful throughput for three coronagraph types: pupil mapping, vortex phase mask, and band-limited Lyot designed for inner working angles of 2 and $4 \lambda / \mathrm{D}$ as indicated. Our analysis will consider this and other metrics of science performance in the presence of real-world optical wavefront errors.

We will use metrics for coronagraph performance characteristics that are critical to the ACCESS science program including achievable contrast, spectral bandwidth, and extent of the high-contrast discovery area. One such metric is "useful throughput" ${ }^{10}$ of an ideal system. We have computed this metric for an example of three of the coronagraph types: (1) fourth-order hybrid band-limited Lyot $^{11}$ designed for 2 and $4 \lambda / \mathrm{D}$ inner working angles; (2) pupil mapping with a new apodization scheme ${ }^{12}$; and (3) fourth-order vector vortex phase mask ${ }^{13}$. We find that these coronagraph types are each capable, in principle, of $\mathrm{C}=10^{-10}$ contrast imaging at angular separations of $2 \lambda / \mathrm{D}$.

To be meaningful, estimates of coronagraph performance require accurate prediction of real-world wavefront errors. Each of the coronagraph types carry starlight through various optical elements to a "dark spot" where the bulk of the starlight is removed from the system. For the band-limited and phase mask coronagraphs with two DMs, there are $\mathrm{N}=6$ critical elements between the telescope focal point and a hard-edged pupil (Lyot) stop. Similarly, there are $N=5$ elements for the shaped pupil and $\mathrm{N}=10$ elements for the pupil mapping approach between first focus and a hard-edged field stop. These are the critical components for suppression of starlight and preservation of high contrast. The achievable contrast, inner working angle (IWA), and throughput are sensitive to surface figure errors and reflectance irregularities on the mirrors, optical misalignments, telescope pointing jitter, thermal drift, scattered light from dust and surface defects, stray light, and cross-polarization effects from off-axis mirrors. The ACCESS study will establish a model for the baseline optical system and uniformly apply these irregularities as perturbations to predict their effects on science performance for each coronagraph type.

We adopt "simplicity" as a design principle for the coronagraph systems. The number of critical coronagraph elements $\mathrm{N}$ is one metric of instrument complexity, representing the buildup of wavefront errors, cost of component manufacturing and 
testing, overall throughput loss, potential failure points, and the risk of schedule delays as the component count is increased. Further, the number of possible interactions between critical elements, $\mathrm{K}=\mathrm{N}(\mathrm{N}-1) / 2$, drives the complexity of end-to-end contrast modeling and analysis, sensitivity to optical misalignments, accumulation of stray light within the instrument, and the extent and cost of subsystem performance verification tests at all stages of development. ACCESS will carry out a riskbenefit analysis among the coronagraph types, to achieve the best possible science within the resource limits of a medium scale mission.

\subsection{The observatory}

We baseline an unobstructed off-axis Gregorian telescope with a $1.5 \mathrm{~m}$ primary mirror. Cost studies indicate that a 1.5meter primary mirror is near the upper limit in mirror size for a medium-class mission concept. It will be finished to smoothness of better than $5 \mathrm{~nm} \mathrm{rms}$, similar to HST, in the critical mid-spatial-frequency $(5-50 \mathrm{~cm} / \mathrm{cycle})$ range that scatters light within the first few arcseconds of the star. The Gregorian telescope provides a focal point between the primary and secondary mirrors, an advantageous location to place a baffle to help isolate the secondary mirror and the rest of the optical system from stray light and external thermal inputs. The secondary mirror assembly is supported by a composite forward metering shell. A temperature-controlled outer barrel assembly surrounds the entire assembly to provide the required thermal stability.

The spacecraft is based on Northrop Grumman's T310 spacecraft bus, adapted for operation in a halo orbit around the second Sun-Earth Lagrange point (L2) and launch in an Atlas 401 vehicle.

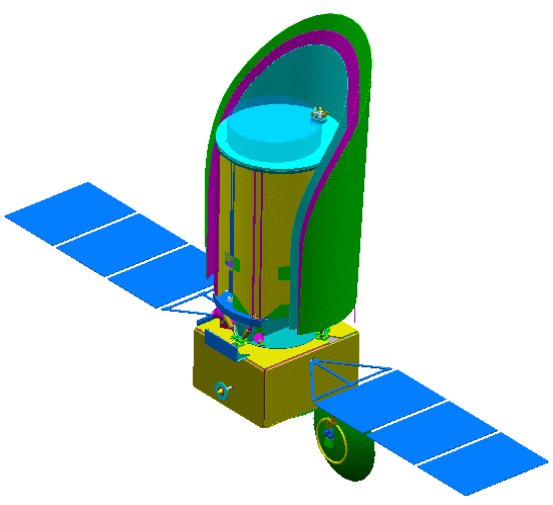

Figure 3. The ACCESS observatory. Telescope, surrounded by a multilayer sunshade, is attached to the spacecraft configured for operations at L2. The coronagraph is located between the telescope and spacecraft.

The spacecraft provides payload platforms, equipment mounting panels, solar arrays, star trackers, propulsion modules, and thermal control elements. The attitude of the spacecraft is maintained by reaction wheels, using a hierarchy of knowledge from a coarse sun sensor, a pair of star trackers, and pointing knowledge from the coronagraph. The propulsion system is provided for L2 orbit insertion, station keeping, reaction wheel momentum dumping, and attitude control during contingency operations. The temperature of the payload and spacecraft components is maintained by a thermal control subsystem, including a surrounding multilayer sunshade.

\subsection{Systems: Pointing control and thermal stability}

Wavefront stability is paramount for the telescope system, and will be studied in detail for ACCESS concept. Drift in the alignments in the optical system will degrade contrast performance and drive requirements for periodic tuning of the wavefront control system. Thermal gradients in the telescope metering structure could be a source of alignment drift, and gradients across the primary mirror could be the dominant source of low-spatial-frequency distortions in the wavefront. It is important to model and design the thermal control system with sufficient fidelity that the coronagraph and telescope optics can be stabilized to the required levels over periods of hours. This requires a systems approach that integrates the telescope architecture, proven materials, and dynamic and thermal control. The required structural stability is achieved with ULE glass, Invar, and composite materials for low coefficients of thermal and moisture expansion. The relative alignment of the primary and secondary mirrors will be held stable to the 0.5 -micron level, while the surface figure of the primary mirror is held stable to the $1 \mathrm{~nm}$-rms level over the mid-spatial-frequency range.

The achievable contrast at the inner working angle is directly dependent on the ability of the pointing control system to stabilize the image of the bright central star. The pointing of the observatory must be actively stabilized by a precise 
attitude control system (ACS). The baseline ACS relies on the spacecraft to keep body pointing errors within achievable limits, while a fine steering mirror located at a pupil within the coronagraph maintains precise centration of the star. An active jitter isolation system for the telescope-spacecraft interface will be studied for enhanced control of pointing jitter. Adequacy and enhancements of the pointing control system, as required by science objectives and consistent with the scope of a medium-class mission, will be evaluated during the concept study.

\subsection{The four coronagraph types}

While noting that our ultimate goal is an optimal synthesis of coronagraph elements, we emphasize that recentlypublished developments in the field already provide examples of value of the hybrid approach, since each one of them already benefits from a combination of amplitude and phase control. A current summary of the status of coronagraph technologies can be found in the "Coronagraph Workshop 2006" proceedings ${ }^{14}$.

The band-limited Lyot coronagraph is the most studied of the four types. The concept was introduced by Kuchner and Traub $^{15}$ and extended to higher-order configurations by Kuchner et al. ${ }^{16}$. These coronagraph masks have been manufactured in HEBS glass ${ }^{17}$ and more recently as vacuum-deposited metal films ${ }^{11}$. The best high-contrast results to date have been obtained with a band-limited coronagraph in JPL's High Contrast Imaging Testbed (HCIT) ${ }^{18}$. Over the last five years, the testbed has steadily set new records in high-contrast imaging, reaching $6.4 \times 10^{-10}$ contrast in a spectral bandwidth of $10 \%$ over a field of view spanning $4-10 \lambda / \mathrm{D}^{11,19}$. The testbed has also been used to demonstrate long-term contrast stability of $10^{-11}$ over periods of five hours or more ${ }^{20}$. Recent work ${ }^{11}$ has shown that hybrid metaldielectric masks, band-limited in both real and imaginary parts, can achieve high contrast over bandwidths of $20-30 \%$ with useful throughputs of order $67 \%$, and representative hybrid masks will be fabricated and tested on the HCIT this year. Analysis has shown that a trade can be made between throughput efficiency and inner working angle, and that IWAs as small as $2 \lambda / \mathrm{D}$ are feasible,

The shaped pupil coronagraph, introduced by Spergel $^{21}$ and developed at Princeton ${ }^{22}$, has also received detailed engineering analysis and has been tested at JPL's HCIT. Pupil masks avoid chromatic effects in wavefront phase, a potential advantage for wide spectral bandwidths. Physical masks have been manufactured from silicon wafers at JPL's Micro Devices Laboratory. The "Ripple 3" design has achieved $2 \times 10^{-9}$ contrast in a spectral bandwidth of $10 \%$ over a field of view spanning $4-10 \lambda / \mathrm{D}^{23}$. The shaped pupil masks to date have been designed specifically for an inner working angle of $4 \lambda / \mathrm{D}$, where a hard-edged focal plane mask is required to block the direct light from the star. Recent investigations ${ }^{24}$ demonstrate the benefits of using a pair of deformable mirrors in conjunction with the shaped pupil to improve coronagraph IWA to $2.3 \lambda / \mathrm{D}$ and throughput to $40 \%$.

The pupil-mapping system, introduced by Guyon et al. ${ }^{25}$, also known as phase induced amplitude apodization (PIAA), uses a pair of steeply aspheric mirrors to concentrate the light towards the center of the coronagraph pupil. PIAA can in principle achieve high contrast and high throughput at inner working angles of $2 \lambda / \mathrm{D}$. The best reported laboratory performance with the first generation of PIAA mirrors is a contrast level of $6.5 \times 10^{-7}$ between $2-4 \lambda / \mathrm{D}$ in monochromatic light ${ }^{12}$. Recent work ${ }^{25,26}$ has shown that additional grey-scale or binary apodizations are needed to improve bandwidth and contrast, leading to hybrid designs that achieve bandwidths of $20 \%$ and throughputs of order $85 \%$. A second generation of PIAA mirrors is now being fabricated for laboratory demonstrations and is scheduled for tests in the HCIT later this year. Studies have been made of the performance of the pupil-mapping coronagraph with one or two deformable mirrors ${ }^{27}$. A pupil-mapped system is more sensitive to low order aberrations than either a Lyot or shaped pupil coronagraph and that, for reasonable levels of dynamic instability, the IWA advantage may be diminished ${ }^{26}$.

The phase mask coronagraph has been developed in a number of forms, including a family of vector vortex masks ${ }^{13}$ and the optical vortex mask ${ }^{28}$. Our main interest lies in the performance and readiness of phase vortex masks designed for topological charge of 4 or greater. Also to be considered are "achromatic chessboard masks" ${ }^{29}$, a higher-order version of the four-quadrant phase mask ${ }^{30}$ which has been used for ground-based observations ${ }^{31}$ and will be used in the MIRI coronagraph on JWST ${ }^{32}$.

Finally, we note that the forgoing coronagraph designs provide the elements of a hybrid coronagraph approach that we will explore by conceptually rearranging the "players" on our "level playing field". Recently-demonstrated methods for the manufacturing of both attenuating and phase-shifting masks raise the possibility of hybrid masks for use at focal planes, pupil planes, or elsewhere in the optical system. The pair of DMs provided in our baseline coronagraph can be used to correct for optical defects and fundamental limitations in the coronagraph architecture, but can also be used to improve bandwidth and contrast beyond the limits of otherwise "pure" coronagraph architectures. 


\subsection{Active wavefront control}

Active control of the optical wavefront is required for our desired coronagraph system. Coronagraph elements are designed to suppress the diffracted light that is present in all telescopes, in some cases achieving in principle perfect suppression. While ideal coronagraphs perform well in simulations with a mathematically perfect wavefront, it would be unrealistic to base design decisions on ideal theoretical performance alone. Light scattered by mirror surface irregularities establishes a contrast floor that cannot be overcome by coronagraphic techniques alone. As a rule of thumb, a wavefront corrected at $\mathrm{n}$ locations across the aperture diameter (e.g. using a DM that maps $\pi \mathrm{n}^{2} / 4$ actuators across the primary mirror) yields a contrast floor $\mathrm{C}=\pi(2 \pi \sigma)^{2} /(\mathrm{n} \lambda)^{2}$, where $\sigma$ is the rms surface error in the DM actuator settings, assumed random and uncorrelated. For example, with $n=64$ (3217 actuators total within a circular stop) for each of two DMs, $\sigma=0.04 \mathrm{~nm} \mathrm{rms}$ is required for an instrument contrast $\mathrm{C}=10^{-10}$ at a wavelength of $500 \mathrm{~nm}$. This level of control has been demonstrated in the laboratory ${ }^{20}$.

A number of wavefront sensing and control methods are available and understood from laboratory experiments, each based on only the imagery of a star at the science focal plane. ACCESS uses a pair of deformable mirrors and control algorithms to simultaneously control wavefront amplitude and phase, creating a high-contrast dark field of view as well as increasing spectral bandwidth and reducing the inner working angles. The great advantage of pure focal-plane sensing is that it avoids the problem of non-common-path errors that plague other methods. These include (1) the Gerchberg-Saxton method, which requires as input a set of out-of-focus images of a target star with the coronagraph elements temporarily removed from the beam, used for on-orbit telescope alignment and initial DM settings. And (2) the EFC method ${ }^{33,34}$, that uses deterministic settings of the DMs to probe the complex (amplitude and phase) wavefront and iteratively reduce the energy scattered within the coronagraph dark field.

The deformable mirrors baselined for ACCESS are an outgrowth of nine years of development at Xinetics and JPL. A modular actuator array is based on PMN electroceramic actuator materials widely used in adaptive optics systems. Manufactured arrays, sized $32 \times 32$ and $48 \times 48 \mathrm{~mm}$, provide 1024 and 2304 actuators respectively per module. The DM assembly includes a continuous fused silica facesheet and electrical connections to a multiplexed driver system. These modules can be bonded together to form larger arrays, e.g., a bonded assembly of four $32 \times 32$ actuator modules with a single facesheet forms a $64 \times 64 \mathrm{~mm}$ DM with 4096 actuators, of the type now available for ACCESS. DMs of this type have been successfully used in a flight-like vacuum environment in laboratories at JPL for over 5 years. Thermal cycles during the manufacture of the DMs reach $80^{\circ} \mathrm{C}$ or higher during final assembly, indicating tolerance to a wide range of operational temperatures. Shake tests on engineering prototypes of these DMs, at levels representative of the launch in a Delta 2 vehicle, have established the basic ability of the Xinetics mirrors to survive launch loads.

\subsection{Focal plane sensors and spectral discrimination}

The ACCESS focal plane will be used for simultaneous imaging in three $20 \%$ photometric bands and for integral field spectroscopy at nominal resolutions of $\mathrm{R}=20$. In addition, the focal plane provides images needed for telescope alignment, initial acquisition of target stars, and periodic on-orbit instrument calibration.

We anticipate that each of the coronagraph types can provide images in $20 \%$ passbands that cover the visible spectrum, e.g. 500-600 nm, 600-720 nm, and 720-870 nm. The high-contrast image can be split with dichroic mirrors into three spectral bands, as suggested in Figure 4 for the case of a Lyot coronagraph. Variations on this theme will be devised to accommodate the unique requirements of each coronagraph type. The shortest wavelength image will necessarily provide the smallest $\lambda / \mathrm{D}$ and IWA, hence the greatest sensitivity to planetary objects in the field. The longer wavelength images provide a tool for discrimination between astronomical objects and instrumental speckles in the field, and with additional integration time will provide follow-up spectrophotometry on interesting exoplanetary systems. A commercially-available low-noise CCD is used for this imaging mode. 


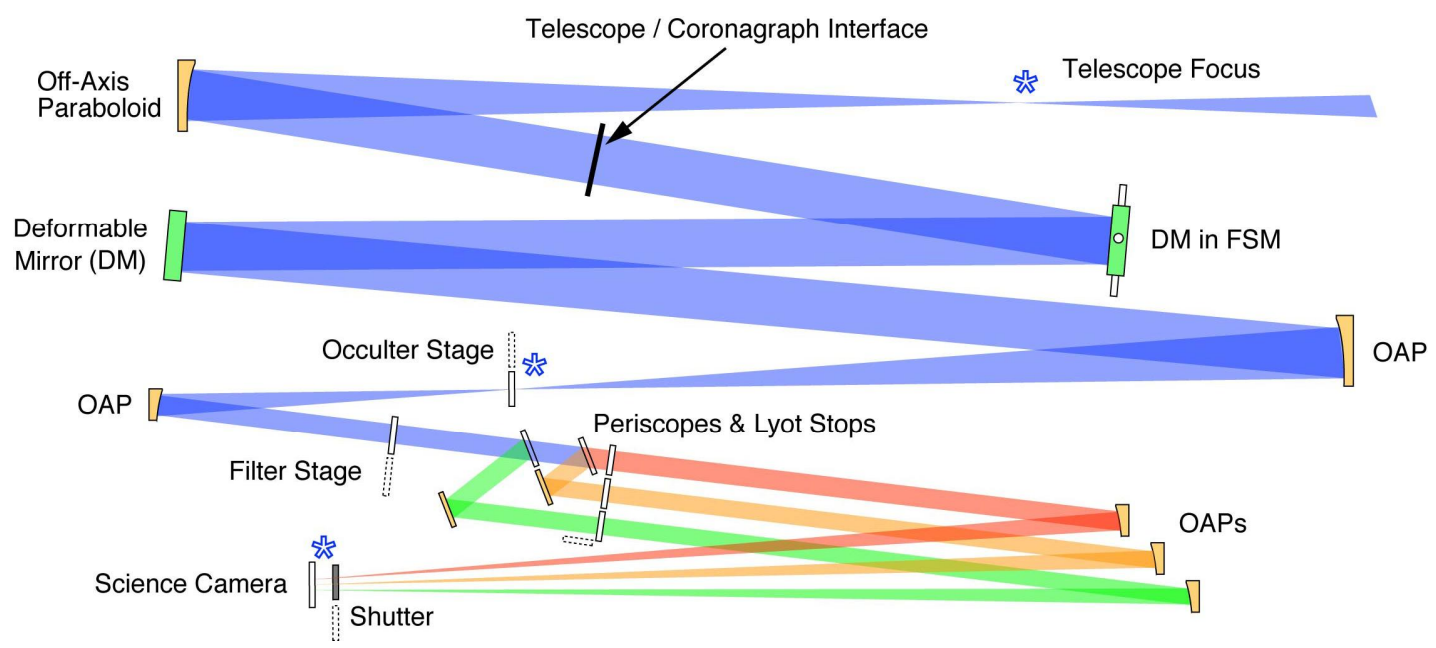

Figure 4. One of a number of candidate Lyot coronagraph layouts to image simultaneously in three spectral bands with a single CCD. Variations on this basic theme will be devised for each of the coronagraph types, using the same instrument elements as much as possible.

ACCESS will include an integral field spectroscopic mode with access to all three spectral bands. A flip mirror located just before the coronagraph focal plane and relay optics direct a selected $10 \times 10 \lambda / \mathrm{D}$ area of interest within the coronagraph dark field to a lenslet/pinhole array, followed by a diffraction grating, lenses, and a low-read-noise CCD comprise the integral field unit. The background noise, once the spectra are dispersed at $\mathrm{R}=20$, would be dominated by the read noise of conventional CCD detectors. In the last five years, electron-multiplying CCDs with gain from multistage electron impact ionization have been available with small but increasing formats, now up to $1 \mathrm{k} \times 1 \mathrm{k}$ pixels for the e2v L3CCD. A signal discriminator enables these detectors to become photon-counting imagers. Readiness of this technology will be evaluated during the study.

\section{CONCEPT STUDY}

The ACCESS study is organized in two phases. The first phase defines a point design for the observatory system, from which reliable estimates can be made for the accuracy of pointing control and the thermal and dynamic stability of the telescope system. This information will be used to predict the consequences for performance of the optical system that will be used in subsequent coronagraph trades. In parallel, also in the first phase, we will implement a closely-related family of candidate coronagraph layouts using the same pair of deformable mirrors, the same focusing mirrors, and as much as possible a common optical system in preparation for sensitivity analysis of the coronagraphs. A matrix of performance metrics will be developed for each of the four types, and the candidate coronagraph types will be brought to a comparable level of tolerancing. This sets the stage for phase two.In the second phase, existing Fresnel propagation and science simulation codes will be used to estimate performance metrics and science reach of the candidate coronagraph systems. This will lead to a review of the baseline definition and to a revised point-design. The second phase continues with analysis of the coronagraph performance and definition of the 5-year science mission.

\subsection{Performance models}

A detailed optical model of the baseline observatory has been developed and validated by experiment, and will be further refined in the course of this study, ultimately to form the basis of a predictive model for the proposed mission concept. The coronagraph model, which has been validated with laboratory testbed measurements, incorporates the expected surface figure errors of commercially available mirrors, the complex (amplitude and phase) characteristics of the coronagraph masks, and the measured properties of the DMs. End-to-end Fresnel diffractive propagation analysis is carried out using a various software packages, including the publicly-distributed PROPER package ${ }^{35}$. Polarization properties will be analyzed, including the characteristics of protected silver mirror coatings in an off-axis telescope system.

A comparison matrix will be compiled, to include IWA, contrast, spectral bandwidth, throughput efficiency, complexity, TRL, and cost for each coronagraph. ACCESS will use validated optical propagation models to compute these metrics on the "level playing field" of the baseline observatory, as predicted with optical, thermal, and structures modeling for the spacecraft, telescope, and coronagraph systems. 


\section{SUMMARY}

ACCESS is one of four medium-class exoplanet concepts selected for the NASA Astrophysics Strategic Mission Concept Study (ASMCS) program. Its science objective is the direct imaging and spectroscopic exploration of the nearby exoplanet systems, including the observation of major planets, super-earths, dust and debris structures, and possibly a handful of earth-sized planets orbiting the FGK stars within 10-20 pc. The study compares science performance and readiness of four major coronagraph categories.

ACCESS will compare the four major coronagraph types on a "level playing field" in terms of a conceptual space observatory. The conceptual observatory includes the telescope, spacecraft, pointing control system, thermal control system, and wavefront sensing and control hardware and algorithms representative of the current state-of-the-art for a medium-class NASA mission, with all observatory elements common to the four coronagraph types.

The ACCESS study will then evaluate the relative merits and readiness of the four major coronagraph types, as well as hybrid combinations. To the extent possible, the best current example of each of the four coronagraphs will be demonstratred in the HCIT during the year of the study, again on a level experimental playing field that includes a spacelike vacuum environment and a state-of-the-art wavefront control system that will be common to all four coronagraph types.

The ACCESS science program will be further defined and readiness for the mission will be evaluated in terms of the predicted performance of a high-TRL mission architecture. Technologies critical to the baseline ACCESS concept will be reviewed, and opportunities for technology improvements that would better serve the science mission objectives, and may be feasible in the coming 5 years, will be identified. The potential impact of improved technologies will be included as part of the final report.

\section{REFERENCES}

1. Dermott, S.F., K. Grogan, E.K. Holmes, M.C. Wyatt, "Signatures of planets," Exozodiacal Dust Workshop Conference Proceedings, ed. Backman, D.E. et al., NASA/CP-1998-10155, 59 (1988).

2. Kalas, P., J.R. Graham, M. Clampin, "A planetary system as the origin of structure in Fomalhaut's dust belt," Nature 435, 1067 (2005).

3. Schneider, J., et al., "Biosignatures and extrasolar planet characterization: visible versus infrared," in: Proceedings of the conference on Towards Other Earths: DARWIN/TPF and the Search for Extrasolar Terrestrial Planets, Heidelberg, Germany (2003).

4. Bally, J., C.R. O’Dell, M.J. McCaughrean, "Disks, Microjets, Windblown Bubbles, and Outflows in the Orion Nebula," A. J. 119, 2919 (2000).

5. Burrows, C., et al., "Hubble Space Telescope Observations of the Disk and Jet of HH 30," Ap.J. 473, 437 (1996).

6. Krist, J.E., et al., "WFPC2 images of a face-on disk surrounding TW hydrae," Ap.J. 538, 793 (2000).

7. Dullemond, C., C. Dominik, "Flaring vs. self-shadowed disks: The SEDs of Herbig Ae/Be stars," Astron. and Astrophys. 417, 159 (2004).

8. Stern, S.A., J.M. Shull, J.C. Brandt, "Evolution and detectability of comet clouds during post-main-sequence stellar evolution," Nature 345, 305 (1990).

9. Swain, M., G. Vasisht, and G. Tinetti, "The presence of methane in an exosolar planet atmosphere," Nature 452, 329 (2008).

10. Guyon, O., et al., "Theoretical limits on extrasolar terrestrial planet detection with coronagraphs," Ap.J.Suppl. 167, 81 (2006).

11. Moody, D., B. Gordon, and J. Trauger, "Design and demonstration of hybrid Lyot coronagraph masks for improved spectral bandwidth and throughput," Proc. SPIE 7010, 7010-81 (2008).

12. Guyon, O., et al., "Pupil-mapping Exoplanet Coronagraphic Observer (PECO)," Proc. SPIE 7010, 7010-66 (2008).

13. Mawet, D., P. Riaud, O. Absil, J. Surdej, “Annular Groove Phase Mask Coronagraph,” Ap.J. 633, 1191 (2005).

14. Traub, W., editor, "Coronagraph Workshop 2006," http://planetquest.jpl.nasa.gov/TPF/ tpf-c_workshopDocs.cfm (2007).

15. Kuchner, M.J., and W.A. Traub, "A Coronagraph with a Band-limited Mask for Finding Terrestrial Planets," Ap.J. 570,900 (2002).

16. Kuchner, M. J., J. Crepp, J. Ge, "Eighth-order image masks for terrestrial planet finding,” Ap.J. 628, 466 (2005).

17. Wilson, D., P.J. Maker, J. Trauger, T. Hull, "Eclipse apodization: realization of occulting spots and Lyot masks," Proc. SPIE 4860, 361 (2002). 
18. Trauger, J., et al., "Laboratory demonstrations of high-contrast imaging for space coronagraphy," Proc. SPIE 6693, 66930X (2007).

19. Kern et. al., "TPF-C Milestone \#2 Report," in preparation (2008).

20. Trauger, J.T., and W.A. Traub, "A laboratory demonstration of the capability to image an Earth-like extrasolar planet," Nature 446, 771 (2007).

21. Spergel, D.N., "A new pupil for detecting extra-solar planets," astro-ph/0101142 (2000).

22. Kasdin, N.J., R.J. Vanderbei, M.G. Littman, D.N. Spergel, "Optimal one-dimensional apodizations and shaped pupils for planet finding coronagraphy," Applied Optics 44, 1117 (2005).

23. Belikov, R., et al., "Demonstration of high contrast in $10 \%$ broadband light with the shaped pupil coronagraph," Proc. SPIE, 6693, 66930Y (2007).

24. Pueyo, L., R. Belikov, J. Kasdin, R. Vanderbei, "Performance study of integrated coronagraph-adaptive optics designs," Proc. SPIE 6693, 669307 (2007).

25. Guyon, O., et al., "Exoplanet imaging with a Phase-Induced Amplitude Apodization coronagraph. I. Principle," Ap. J. 622, 744 (2005).

26. Belikov, R., N.J. Kasdin, R.J. Vanderbei, "Diffraction-based Sensitivity Analysis of Apodized Pupil-mapping Systems," Ap.J. 652, 833 (2006).

27. Shaklan, S., A. Give'on, R. Belikov, L. Pueyo, O. Guyon, "Broadband wavefront control in a pupil mapping coronagraph,” Proc. SPIE 6693, 66930R (2007).

28. Swartzlander, G., "Peering into darkness with a vortex spatial filter," Opt. Lett. 26, 497 (2001).

29. Rouan, D., D. Pelat, M. Ygouf, "Nulling interferometry - A new concept of achromatic phase shifters using cellular mirrors," Spirit of Lyot Conference, http://astro.berkeley.edu/ kalas/ lyot2007/agenda.html (2007).

30. Rouan, D., et al., “The four-quadrant phase mask. I. Principle,” P.A.S.P. 112, 1479 (2000).

31. Boccaletti, A., et al., "The four-quadrant phase mask coronagraph. IV. First light at the Very Large Telescope," P.A.S.P. 116, 1061 (2004).

32. Baudoz, P., et al., "Feasibility of the four-quadrant phase mask in the mid-infrared on the James Webb Space Telescope," P.A.S.P. 118, 765 (2006).

33. Give'on, A., B. Kern, S. Shaklan, D. Moody, L. Pueyo, "Broadband wavefront correction algorithm for highcontrast imaging systems," Proc. SPIE 6691, 66910A (2007).

34. Borde, P., and W. Traub, "High-contrast imaging from space: speckle nulling in a low aberration regime," Ap.J. 638,488 (2006).

35. Krist, J.E., "PROPER: An Optical Propagation Library for IDL,” Proc. SPIE, 6675, 66750P (2007). 